\title{
The Role of Gut Microbiome in Autoimmune Uveitis
}

\author{
Xiangyu Fu ${ }^{a, b}$ Yongjiang Chen ${ }^{c}$ Danian Chen ${ }^{a, b}$ \\ aThe Research Laboratory of Ophthalmology and Vision Sciences, State Key Laboratory of Biotherapy, West China \\ Hospital, Sichuan University, Chengdu, China; ${ }^{\mathrm{b} T}$ The Department of Ophthalmology, West China Hospital, Sichuan \\ University, Chengdu, China; 'The School of Optometry and Vision Science, University of Waterloo, Waterloo, ON, \\ Canada
}

\section{Keywords}

Gut microbiome · Autoimmune uveitis · Antigenic mimicry · Metabolism · Therapy

\begin{abstract}
The gut microbiome has important physiological functions and plays an indispensable role in the human body. Currently, there are an increasing number of studies revealing the close correlation between dysbiosis of the gut microbiome and a variety of autoimmune diseases, including autoimmune uveitis. This brief review summarizes recent literature regarding the relationship between dysbiosis and the occurrence and development of autoimmune uveitis. Dysbiosis participates in the pathogenesis of autoimmune uveitis largely by 4 mechanisms: antigenic mimicry, disturbance of intestinal immune homeostasis, destruction of the intestinal barrier, and reduction of beneficial anti-inflammatory metabolites. Further elucidation of these mechanisms will facilitate the treatment of the gut-microbiome-relevant autoimmune diseases by potential therapeutic strategies, such as antibiotics, probiotics, diet modifications, and fecal microbial transplantation.

(c) 2020 S. Karger AG, Basel
\end{abstract}

karger@karger.com

(C) 2020 S. Karger AG, Base

www.karger.com/ore

Karger!

\section{Introduction}

Uveitis, an inflammatory disease of the intraocular uveal tissues (including the iris, ciliary body, and choroid), can cause serious visual impairment and may also involve adjacent tissues such as the retina, optic nerve, and vitreous body. Uveitis often occurs in young adults; the median age of onset of uveitis is around 35 years. Uveitis accounts for about a quarter of all cases of blindness in developing countries and for $10-15 \%$ of preventable cases of blindness in developed countries $[1,2]$. About $35 \%$ of all uveitis patients have been reported to suffer significant visual impairment or legal blindness. Prompt therapy and rapid control of ocular inflammation are integral to maintaining good visual acuity. At present, the mainstay treatment of uveitis involves the use of ciliary muscle paralysis agents and glucocorticoids (both locally and systemically). Addition of immunomodulatory therapy increases disease remission and limits steroid use. Depending upon its etiology, uveitis may be classified into infectious or noninfectious uveitis; the latter is primarily related to autoimmunity (autoimmune uveitis) [3]. Autoimmune uveitis often comorbid with other autoimmune diseases, such as ankylosing spondylitis, or is 
the ocular manifestation of systemic immune diseases such as systemic sarcoidosis, Behcet's disease (BD), and Vogt-Koyanagi-Harada (VKH) disease $[4,5]$. An imbalance between autoreactive pathogenic effector T-cells, including T helper 1 (Th1) and Th17 lymphocytes, and regulatory T cells (Tregs), has been implicated in the pathogenesis of autoimmune uveitis. However, the exact mechanism for pathogenesis of autoimmune uveitis remains unclear [6]. Recent studies suggest causative roles of gut microbiota in the development of autoimmune diseases, including uveitis [7].

The microbiota is defined as microorganisms in a specific environment, most commonly the gastrointestinal tract [8]. Gut microbiota refers to all kinds of microorganisms colonizing the intestinal tract, consisting of about $10^{14}$ microorganisms, whose collective genome is $>100$ times the size of the human genome [9]. The populations comprised bacteria, fungi, viruses, protozoa, and Archaea; bacteria are dominant and largely consist of the phyla Firmicutes and Bacteroidetes and a lesser number of Actinobacteria and Proteobacteria $[9,10]$. The definition of the microbiome is somewhat ambiguous. Some define the microbiome narrowly as only the collective genomes of the microbiota [11]; others prefer to apply the term to the sum total of the genes and genomes of the microbiota, as well as the products/metabolites of the microbiota and the host environment [12]. We favor the latter definition of the microbiome in this review.

Gut microbiome has many important physiological functions, such as protecting the host from pathogens, promoting digestion and absorption, regulating the function of the immune system, synthesizing vitamins and amino acids, and metabolizing drugs [13]; thus, it is considered an indispensable part of the human body. The microbiome evolves within a healthy host from birth to death, constantly fine-tuning itself to maintain a homeostatic balance with the host's immune system. The evolution of the human microbiome is governed by host factors such as both the adaptive and the innate immune system, as well as external factors such as diet, medication and toxin exposure, and illness [14]. An imbalance of the normal gut microbiota composition is called dysbiosis [15]. Dysbiotic conditions can favor invasion and growth of pathogenic species and can disrupt the finely tuned regulatory circuits of the immune system, thus potentially induce diseases [14]. Indeed, an increasing number of studies have demonstrated that dysbiosis may be closely connected to a variety of diseases, including autoimmune diseases (e.g., ankylosing spondylitis [16, 17], rheumatoid arthritis [18], and multiple sclerosis $[19,20])$, inflamma-

Gut Microbiome and Autoimmune

Uveitis tory diseases (e.g., inflammatory bowel disease $[18,21]$ ), neurological diseases (e.g., Alzheimer's disease $[9,20]$ and Parkinson's disease [22]), and eye diseases (e.g., agerelated macular degeneration $[23,24]$, diabetic retinopathy [25], dry eye [26, 27], and glaucoma [28, 29]). The purpose of this review is to explore the role and mechanism of the gut microbiome in autoimmune uveitis and to propose several potentially effective therapeutic strategies to treat uveitis by targeting gut microbiome.

\section{Correlation between the Gut Microbiome and Autoimmune Uveitis}

Over the past 2 decades, experimental data have demonstrated the critical role of the intestinal microbiome in controlling both innate and adaptive immune responses. The strongest evidence of the role of the gut microbiota in disease pathogenesis comes from studies using germfree mouse models of human autoimmune diseases, including spontaneous ankylosing enteropathy [30], type 1 diabetes [31], autoimmune arthritis [32], and experimental autoimmune encephalomyelitis (EAE; an animal model for multiple sclerosis) [33]. In most of these disease models, the severity and/or incidence of disease are reduced under germ-free conditions consistent with the microbiota being a trigger for disease progression [34, 35]. The notion that dysbiosis may be closely connected to autoimmune diseases is also supported by clinical investigations of ankylosing spondylitis [16, 17], rheumatoid arthritis [18], and multiple sclerosis [19, 20]. Although uveitis is an autoimmune disease, the correlation between the intestine microbiome and uveitis was only gradually established within the last 5-6 years, based on both experimental studies using animal models and clinical observations (shown in Table 1), and further supported by findings that common immunosuppressant drugs used in the treatment of autoimmune diseases have some anti-microbial properties.

It was demonstrated that depleting the gut microbiota attenuates disease severity in mouse models of autoimmune uveitis [36-38]. Two mouse models of experimental autoimmune uveitis (EAU), induced EAU and spontaneous EAU, were used in these studies. Induced EAU is established by active immunization with the retinal protein inter-photoreceptor retinoid-binding protein (IRBP) emulsified in complete Freund's adjuvant, a mixture of mineral oil with heat-killed Mycobacterium tuberculosis. Pertussis toxin is given as an additional stimulus in C57BL/6 mice to facilitate disease induction. Induced 
Table 1. Major references connecting the gut microbiome with uveitis

\begin{tabular}{|c|c|c|c|c|}
\hline Year & Study type & Subjects & Major findings & Ref. \\
\hline 2015 & Experimental & R161H/B10.RIII & GF/broad-spectrum antibiotics reduced EAU & [38] \\
\hline 2016 & Experimental & C57BL/6J EAU & GF/broad-spectrum antibiotics reduced EAU & {$[36]$} \\
\hline 2017 & Experimental & C57BL/6J EAU & IRT-5 probiotics reduced EAU & {$[39]$} \\
\hline 2019 & Experimental & B10.RIII EAU & Intestinal permeability increased in EAU & {$[67]$} \\
\hline 2016 & Clinical & $12 \mathrm{BD} / 12 \mathrm{HC}$ & $\begin{array}{l}\text { Bifidobacterium and Eggerthella increased and Megamonas and Prevotella decreased } \\
\text { in BD }\end{array}$ & {$[4]$} \\
\hline 2018 & Clinical & 13 uveitis/13 HC & $\begin{array}{l}\text { Faecalibacterium, Bacteroides, Lachnospira, and Ruminococcus reduced and } \\
\text { Prevotella copri and Streptococcus enriched in uveitis }\end{array}$ & {$[43]$} \\
\hline 2018 & Clinical & $38 \mathrm{AAU} / 40 \mathrm{HC}$ & 7 fecal metabolites increased in AAU & {$[40]$} \\
\hline 2019 & Clinical & 14 uveitis/24 HC & Gut fungal richness and diversity decreased and pathogenic fungi enriched in uveitis & {$[44]$} \\
\hline 2020 & Clinical & $82 \mathrm{VKH} / 63 \mathrm{HC}$ & $\begin{array}{l}\text { Butyrate-producing bacteria, lactate-producing bacteria, and methanogens depleted } \\
\text { and Gram-negative bacteria enriched in VKH }\end{array}$ & {$[42]$} \\
\hline
\end{tabular}

GF, germ-free; EAU, experimental autoimmune uveitis; SCFAs, short-chain fatty acids; BD, Behcet's disease; HC, healthy controls; AAU, acute anterior uveitis; VKH, Vogt-Koyanagi-Harada.

EAU models are generally made in B10.RIII or C57BL/6J mice [5]. The spontaneously uveitic R161H mice express an IRBP-specific T-cell receptor (TCR) transgene on the uveitis-susceptible B10.RIII background. R161H mice have an expanded peripheral population of $\mathrm{CD} 4^{+} \mathrm{T}$ cells specific for IRBP 161-180 peptide and spontaneously develop autoimmune uveitis [5].

It was shown that induced EAU in conventionally housed mice is much more severe than that in germ-free C57BL/6J mice. Germ-free mice had lower numbers of infiltrating macrophages, significantly less T-cell infiltration in the retina, as well as reduced numbers of IFN- $\gamma$ and IL-17-producing T cells and increased numbers of regulatory $\mathrm{T}$ cells in the eye-draining lymph nodes, suggesting a close association of gut microbiome with EAU [36]. Oral, but not intraperitoneal, treatment of broadspectrum antibiotics (metronidazole and vancomycin) significantly reduced clinical scores of induced EAU in B10.RIII mice [37]. Oral metronidazole and ciprofloxacin also reduced clinical scores of induced EAU in C57BL/6J mice [36]. These results confirmed the contribution of gut microbiota in the EAU model. Oral gavage feeding of
IRT-5 probiotics (consisting of Lactobacillus casei, Lactobacillus acidophilus, Lactobacillus reuteri, Bifidobacterium bifidum, and Streptococcus thermophilus) also reduced clinical scores of induced EAU in C57BL/6J mice, supporting that gut microbiota may modulate clinical manifestations of EAU [39]. In addition to these studies based on the classic IRBP-induced EAU model, studies in the $\mathrm{R} 161 \mathrm{H}$ spontaneous-EAU model have also suggested a role for the microbiome in the pathogenesis of uveitis. Both rearing $\mathrm{R} 161 \mathrm{H}$ mice under germ-free conditions and treating them prior to birth with a combination of broad-spectrum antibiotics (ampicillin, metronidazole, neomycin, and vancomycin) resulted in significant attenuation of spontaneous disease and was associated with decreased frequencies of IRBP-specific T cells in the intestinal lamina propria [38].

There are also several clinical observational studies from Asian countries (Japan, China, and India), comparing the microbiota of uveitis patients with healthy controls, which collectively support conclusions drawn from EAU animal studies. Shimizu et al. [4] studied the gut microbiota of $12 \mathrm{BD}$ patients with uveitis and 12 unaffected 
individuals in Japan. They reported increased Bifidobacterium and Eggerthella and decreased Megamonas and Prevotella in $\mathrm{BD}$ patients, suggesting the dysbiosis may have an association with the pathophysiology of BD [4].

Huang et al. [40] studied the microbiota composition and fecal metabolites in Chinese patients with acute anterior uveitis (AAU) compared with healthy volunteers. They did not identify a difference in gut microbiota composition, but did show that the fecal metabolic phenotype in AAU patients was significantly different from healthy controls. The expression of 7 fecal metabolites (including 6-deoxy-D-glucose 1 , linoleic acid, $N$-acetyl-beta-D-mannosamine 3 , shikimic acid, azelaic acid, isomaltose 1 , and palmitoleic acid) was increased in AAU patients [40]. This group also showed that fecal microbial transplantation (FMT) from patients with BD [41] or active VKH disease [42] into autoimmune uveitis-prone B10.RIII mice significantly exacerbated EAU activity and increased the production of inflammatory cytokines including IL17 and IFN- $\gamma$. These results suggest potential causation between intestinal dysbiosis and noninfectious uveitis. They also reported relative enrichment of Gram-negative bacteria (Paraprevotella spp.) and depletion of butyrateproducing bacteria, lactate-producing bacteria, and methanogens in the active VKH patients compared with the control group. These differences were reduced after an immunosuppressive treatment. The presence of $\mathrm{Bac}$ teroides sp.2.1.33B, Paraprevotella clara, Alistipes finegoldii, and Eubacterium eligens was associated with good response to immunosuppressive therapy. Two microbial marker profiles were established to distinguish VKH patients from healthy controls and to predict the effectiveness of treatment, respectively [42].

Chakravarthy et al. [43] studied the gut microbiota of 13 Indian uveitis patients (including idiopathic and autoimmune uveitis) and 13 healthy controls with similar ethnic and dietary attributes. They reported decreased microbiota diversity and abundance in their uveitis cohort [43]. Notably, they also demonstrated a reduction in the diversity of several anti-inflammatory microbes (including Faecalibacterium, Bacteroides, Lachnospira, and Ruminococcus) and an increase in the proinflammatory Prevotella copri and pathogenic bacteria Streptococcus in uveitis patients [43]. This group also investigated the gut fungal microbiome within this cohort of uveitis patients and similarly observed a decrease in the number of fungal species and diversity index (including Shannon diversity index, Simpson index, the number of OTUs observed, and Chaol index) in uveitis patients compared with healthy controls [44]. Similar to changes in bacterial mi- crobiota in uveitis patients, most of the fungal genera enriched in the uveitis patients were opportunistic pathogens. In contrast, the fungal genera enriched in the healthy controls included several genera with anti-inflammatory or anti-pathogenic properties [44]. However, it was not specified whether and at what dosages these patients were taking corticosteroids or immunosuppressives.

The dysbiosis of the VKH patients can be partially resolved by an immunosuppressive treatment, suggesting immunosuppressant drugs may have a direct effect on microbiota [42]. Disease-modifying anti-rheumatic drugs (DMARDs) are a class of drugs indicated for the treatment of rheumatoid arthritis and many other autoimmune disorders including uveitis; DMARDs currently include azathioprine (AZA), methotrexate (MTX), cyclosporine A (CsA), and mycophenolate mofetil (MMF) [45]. All 4 of these DMARDs have both immunosuppressive and anti-microbial properties [46]. AZA is an immunosuppressant agent widely used in the management of inflammatory bowel diseases (IBD) and uveitis [47, 48]. AZA is metabolized in the liver to its active form 6-mercaptopurine, which inhibits maturation of B and T lymphocytes through its activity as an antagonist of purine metabolism, resulting in the inhibition of DNA, RNA, and consequently protein synthesis. AZA can suppress the growth of some bacteria such as Campylobacter concisus, Escherichia coli, and Mycobacterium avium paratuberculosis and affect the secretion of extracellular matrix of $E$. coli to inhibit the formation of biofilm $[49,50]$. MTX is a structural analog of folic acid that can competitively inhibit the binding of dihydrofolic acid to the enzyme dihydrofolate reductase, thereby interfering with purine and pyrimidine metabolism and therefore inhibiting DNA and RNA synthesis, DNA repair, and cell division. MTX has been found to be bacteriostatic in vitro against Staphylococcus aureus (but not against E. coli and Pseudomonas aeruginosa) [51].

CsA is a lipophilic cyclic peptide, comprised 11 amino acids and derived from fungi, which selectively inhibits calcineurin, thereby impairing the transcription of IL-2, $\mathrm{TNF} \alpha$, and several other cytokines in T lymphocytes. CsA can disrupt fungal metabolism through calcineurin inhibition and has antifungal activities against Candida albicans and Cryptococcus neoformans. CsA can also inhibit intracellular multiplication of viruses such as hepatitis $\mathrm{C}$ virus and human immunodeficiency virus by binding with cyclophilin [52]. MMF is a selective inhibitor of inosine monophosphate dehydrogenase that interrupts guanosine synthesis. It suppresses T- and B-lymphocyte proliferation, reduces antibody production, and inhibits 
transmigration of leukocytes. MMF has been widely tested for treating refractory uveitis [53]. MMF inhibits the synthesis of microbial DNA and RNA by blocking purine synthesis, thus has a wide-ranging anti-microbial property [46]. The fact that all 4 DMARDs widely used for the management of uveitis also have anti-microbial functions is consistent with the conclusion of EAU studies that depleting the gut microbiota can attenuate EAU severity, thus reinforcing the idea that uveitis is associated with dysbiosis of gut microbiome [36-38].

\section{Mechanisms of Autoimmune Uveitis Caused by Dysbiosis of the Gut Microbiome}

There are a number of related mechanisms involved in the pathogenesis of autoimmune uveitis caused by the dysbiosis, including immune factors (antigenic mimicry, loss of intestinal immune homeostasis, and destruction of the intestinal barrier) and metabolic factors (shown in Fig. 1).

\section{Antigenic Mimicry (Fig. 1a)}

Antigenic mimicry or molecular mimicry is the process whereby autoreactive $\mathrm{T}$ cells are generated through cross-reactivity of gut microbial peptides with self-antigens. It is one of the leading mechanisms by which microbiota may induce autoimmunity $[54,55]$. Microorganisms mimicking protein structures in their host may have an advantage in escaping immune detection. Such similarities may be due to amino acid sequence homology, but also to shared nucleotide sequences or protein structures [56]. Adoptive transfer of spontaneous-EAU $\mathrm{R} 161 \mathrm{H}$ mouse-derived T cells activated in vitro by intestinal contents can induce diseases in many naïve wildtype mice within 6-10 days. This result supports a microbial antigenic mimicry process in the pathogenesis of EAU [38].

Although specific microbial antigens triggering uveitis have yet to be identified, candidate antigens from gut microbiome have been identified for several autoimmune diseases. Neuromyelitis optica (NMO) is a CNS autoimmune disease characterized by immune-mediated demyelination of the optic nerve and spinal cord. Autoreactive humoral and T-cell-mediated immunity against aquaporin 4 (AQP4), a predominant CNS water channel protein, drives NMO pathogenesis. The sequence (p207-216) within the adenosine triphosphate-binding cassette transporter permease (ABC-TP) expressed by Clostridium perfringens has $90 \%$ homology with the 10 -amino acid sequence of AQP4 T-cell epitope p66-75 [57]. The finding that AQP4-specific $\mathrm{T}$ cells in $\mathrm{NMO}$ patients showed cross-reactivity to the ABC-TP protein of the indigenous gut commensal species supports a microbiotarelated molecular mimicry process in NMO pathogenesis $[58,59]$. Microbial cross-reactivity has also been demonstrated in patients with systemic lupus erythematosus. Commensals have been identified in the skin, oral mucosa, and gut of lupus patients and healthy controls that are orthologs of the RNA-binding autoantigen Ro60. Two of these identified commensals, Propionibacterium propionicum and Bacteroides thetaiotaomicron, activated Ro60-specific CD4 ${ }^{+}$memory $\mathrm{T}$ cells from lupus patients $[55,60,61]$. Thus, antigenic mimicry by commensals may be a common trigger of autoimmune diseases including uveitis.

\section{Loss of Intestinal Immune Homeostasis: Imbalance} between Th17 and Tregs (Fig. 1b)

Under normal circumstances, antigen-presenting cells in gut-associated lymphoid tissue (GALT) present potential invasive pathogens to helper $\mathrm{T}$ (Th) cells, especially Th17 cells, to stimulate their proliferation and secrete inflammatory cytokines such as IL-17, which promote the inflammatory response. On the other hand, the presence of Tregs inhibits excessive immune response and prevents persistent inflammation. Th17 cells and Tregs are always in a dynamic balance and together maintain the immune homeostasis of the intestinal tract. Gut commensals can regulate intestinal mucosal immunity, especially the balance between Th17/Tregs [62]. The dysbiosis of gut microbiome could lead to the loss and destruction of intestinal immune homeostasis, lower the threshold of immune activation, break the balance of Th17/Tregs, and result in a significant increase in Th17 generation and a decrease in Tregs production, which contributes to a variety of enteral and extraintestinal inflammatory conditions, such as uveitis [63]. A recent review summarizing the pathogenesis of $\mathrm{BD}$, including infection and genetic and immune factors, showed that alterations of T-cell balance, especially the expansion of Th1 and Th17 cells and the reduction in Tregs were indispensable to the development of BD. Th17 cells and IL-17 pathways were considered to promote the occurrence and activity of the disease [64].

An imbalance between autoreactive pathogenic effector T cells, including Th1 and Th17 lymphocytes with Tregs, has also been implicated in the pathogenesis of autoimmune uveitis [6]. Oral administration of broad-spectrum antibiotics was able to improve the level of Foxp $3^{+}$ Tregs in the lamina propria of the intestine and extraint- 


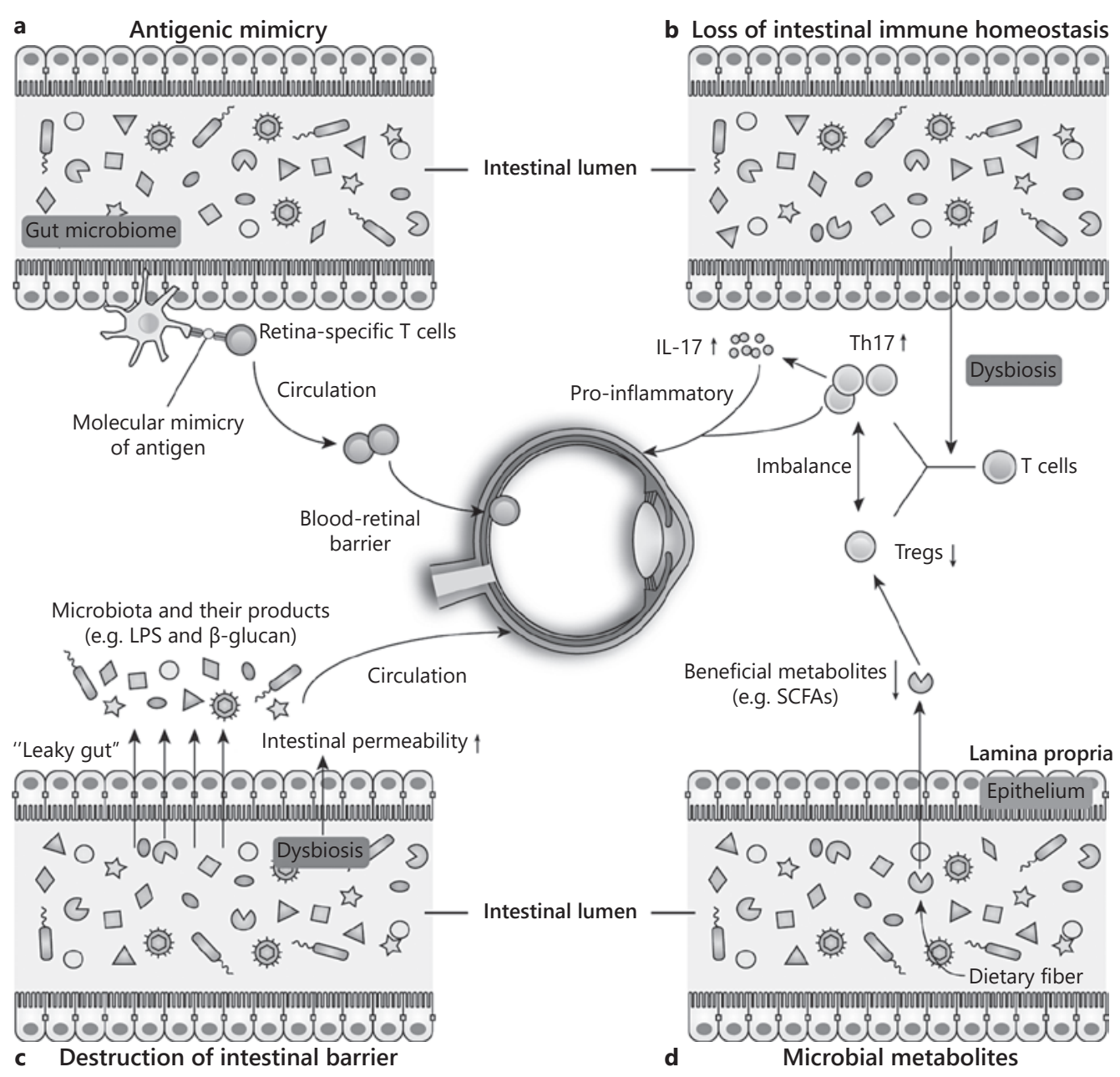

Fig. 1. Four proposed potential mechanisms of autoimmune uveitis caused by dysbiosis of the gut microbiome. a Antigenic mimicry: antigenic mimics in the gut microbiome, which have the highly similar structure or sequence with the autoantigens (e.g., IRBP), could activate retinal-specific $\mathrm{T}$ cells in the periphery. Therefore, the activated retinal-specific $\mathrm{T}$ cells gain the ability to cross the blood-retinal barrier into the eye to induce uveitis. b Loss of intestinal immune homeostasis: the dysbiosis of the gut microbiome could lead to the destruction of intestinal immune homeostasis and break the balance of Th17/Tregs cells in the gut, thus resulting in a significant increase in Th17 cell generation with the increasing secretion of IL-17 and a decrease in Tregs production. The imbalance between Th17 cells and Tregs contributes to uveitis. c Destruction of intestinal barrier: the dysbiosis of the gut microbiome

estinal lymphoid tissues, such as cervical lymph nodes, mesenteric lymph nodes, and spleen, and the proportion of Tregs in the retina also increased, accompanied by the obvious amelioration of inflammatory retinal vascular disruption, while Th1, Th17 cells, and inflammatory cy-

Gut Microbiome and Autoimmune Uveitis could cause intestinal mucosal inflammation and destroy the intestinal barrier, so as to increase intestinal permeability and make the gut leaky. Microbiota and their products (e.g., LPS and $\beta$-glucan) may spread out to the vascular system through the leaky gut, and thus translocate to the eye. If they are trapped or deposited in the uvea, they may become the pathogens and trigger immune responses that cause or exacerbate uveitis. $\mathbf{d}$ Microbial metabolites: intestinal dysbiosis may cause the absence of beneficial microbiota and the reduction in beneficial anti-inflammatory metabolites such as SCFAs, which can in turn increase the production of Tregs, thus indirectly promote the progression of uveitis. IRBP, inter-photoreceptor retinoid-binding protein; SCFA, short-chain fatty acids; LPS, lipopolysaccharide.

tokines decreased, thus reducing the severity of EAU in mice [37]. All of the above indicate that Th17/Tregs imbalance has a pathogenic effect on autoimmune diseases including autoimmune uveitis and the gut microbiome is a potential trigger that should not be ignored. 
Destruction of Intestinal Barrier: Increased Intestinal Permeability (Fig. 1c)

The dysbiosis of the gut microbiome could cause intestinal mucosal inflammation, destroy the intestinal barrier, change intestinal permeability, and contribute to intestinal leakage. Microbiota and their products, such as lipopolysaccharide (LPS) and $\beta$-glucan, can spread to the vascular system and migrate to tissues through a leaky intestinal tract. Once trapped or deposited in tissues such as the synovial membrane or uvea, they may become the pathogens of the diseases (arthritis or uveitis) and trigger immune responses that cause or exacerbate arthritis or uveitis $[65,66]$. For example, increased intestinal permeability has been reported in patients with ankylosing spondylitis, who also showed dysregulation of intestinal tight junction expression and LPS translocation to the vascular system [16].

During the course of an EAU mouse model, there were many morphological changes in intestinal villi, crypt, submucosa, and muscular layer; the ZO- 1 expression and intestinal permeability increased, accompanied by higher expression of anti-microbial peptides such as Reg3 $\gamma$, S100A8, and lipocalin-2 in the intestine, indicating the inflammatory state in the intestine [67]. In addition, the degree of intestinal dysregulation, the increase in permeability, and the elevation of lipocalin- 2 were persistent and in pace with the severity of uveitis. This is consistent with the early hypothesis that intestinal mucosal inflammation may promote the translocation of microbiota or microbial products through increased intestinal permeability, which eventually caused ocular inflammation directly by affecting the eyes or indirectly through molecular mimicry and immune sensitization.

\section{Microbial Metabolites (Fig. 1d)}

Gut microorganisms have the capacity to produce thousands of metabolites, among which short-chain fatty acids (SCFAs), primarily acetic acid, propionic acid, and butyric acid, are the most common. SCFAs are fermented metabolites of dietary fiber produced by microorganisms in the colon, which are beneficial to the body and protective in the animal models of inflammatory disease. It was reported that SCFAs were likely to ameliorate uveitis through 2 mechanisms: one is to increase Tregs in the colon and cervical lymph nodes; the other is to reduce the transport of effector $\mathrm{T}$ cells between the intestine and spleen during uveitis [68]. Exogenous SCFA supplementation decreased the severity of uveitis in mice [68], indicating that intestinal disorders may lead to the absence of some beneficial microorganisms that secrete anti-inflam- matory metabolites such as SCFAs, thus promoting the progression of uveitis. Another study has shown that a unique metabolic phenotype was present in the gut of patients with AAU when compared with healthy controls, and the expression of 7 metabolites (6-deoxy-D-glucose 1 , linoleic acid, $N$-acetyl-beta-D-mannosamine 3 , shikimic acid, azelaic acid, isomaltose 1 , and palmitoleic acid) was significantly elevated in the feces of AAU patients [40]. Previous studies have found that these metabolites were strongly associated with some inflammatory or autoimmune diseases. For example, high levels of $n-6$ fatty acids, particularly linoleic acid, were involved in the pathophysiological process of IBD by stimulating the production of proinflammatory mediators [69]. In nonalcoholic fatty liver disease, derived from the dysregulation of lipid metabolism, linoleic acid and palmitic acid were found to impair mitochondrial function, leading to increased oxidative damage, and mediate selective loss of intrahepatic $\mathrm{CD}^{+}{ }^{+} \mathrm{T}$ lymphocytes, and ultimately accelerate the progression to hepatocellular carcinoma [70]. Azelaic acid, a by-product of fatty acid peroxidation, was reported to promote chronic depression by modulating autoimmune responses [71]. The specific role of these metabolites in AAU is still unclear and warrants further studies. However, it indicates that the importance of metabolic factors in the pathogenesis of autoimmune uveitis should never be overlooked.

It is worth noting that these theories or hypotheses are not mutually exclusive, but explain the pathogenesis of autoimmune uveitis induced by gut microbiome dysbiosis from different aspects (Fig. 1). On the one hand, dysbiosis results in the destruction of intestinal immune homeostasis, the emergence of immune disorders due to decreased immune threshold, and the reduced production of anti-inflammatory metabolites, and the increase in intestinal permeability leads to the translocation of microbiota and microbial metabolites from the intestine into the vascular system; on the other hand, microorganisms could induce uveitis indirectly by the activation of retinalspecific $\mathrm{T}$ cells through antigenic mimicry, or the microbial metabolites may act directly on the eyes to stimulate the development of inflammation. In addition, it has been proposed that extensive and broad extraintestinal migration of immune cells existed [72]. The trafficking of lymphocytes and inflammatory cells from the gut to the eye has been reported in the EAU mice model, where the gutderived cells related to the severity of EAU were detected in the eye [68]. This may be an essential pathogenic mechanism of uveitis, but it still needs to be determined by further studies. 


\section{Therapies Targeting the Gut Microbiome}

Since gut microbiome plays such a vital role in the process of immunity and metabolism during uveitis, therapeutic approaches targeting the gut microbiome to postpone diseases, which have been effective in the treatment of diseases like Clostridium difficile colitis [73], have gradually become a trend. Currently, the main therapeutic strategies contain antibiotics, probiotics, dietary modifications, and FMT.

The role of antibiotics in the treatment for the dysbiosis of the gut microbiome is obvious. As mentioned in previous studies, oral antibiotics were able to reduce the severity of EAU in mice by altering the composition of the gut microbiome, raising the frequency of Tregs in intestinal lamina propria and extraintestinal lymphoid tissues, and decreasing the number of Th1, Th17 cells, and the level of inflammatory cytokines [37]. It has also been reported that oral administration of broad-spectrum antibiotics from 1 week before the immune induction of EAU model could protect mice from severe EAU, akin to germ-free mice, with retinal T-cell infiltration and inflammatory cytokine levels reduced compared with EAU mice [36].

Probiotics are a kind of microbial component with beneficial function, which promote the proliferation of beneficial microorganisms. Oral probiotic strains can regulate immune homeostasis by enhancing Tregs differentiation [74]. As an approach to maintain intestinal health and treat gut microbiome dysbiosis, probiotics are gaining popularity, the importance of which is gradually being noted by researchers. The effects of probiotics on the EAU mouse model was studied, in which mice were pretreated with antibiotics and provided with IRT-5 probiotics, composed of L. casei, L. acidophilus, L. reuteri, B. bifidum, and Streptococcus thermophilus [39]. The results revealed that the application of IRT-5 probiotics might regulate the clinical manifestations of ocular autoimmunity relative to the untreated group to effectively prevent the development of uveitis. Thus, it is possible to deplete gut microbiome with antibiotics in a short time and then recolonize the intestine with beneficial oral probiotics (such as IRT-5). However, the method by which maximizing the colonization of these probiotics in the intestine is a significant obstacle still needs to be overcome.

A high dietary fiber diet is considered to improve the intestinal microecological environment by enhancing the ability of gut microbiome to produce endogenous SCFAs. Dietary modifications have been reported in several ophthalmic diseases, such as diabetic retinopathy and agerelated macular degeneration. It was confirmed that in-

Gut Microbiome and Autoimmune termittent fasting could remodel gut microbiome, improve bile acid metabolism, promote the production of neuroprotective tauroursodeoxycholic acid, which is a secondary bile acid and binds to retinal TGR5 receptors, and ultimately prevent diabetic retinopathy [25]. Lowfat, low-glucose, or low-fructose diets and some micronutrient supplements could also delay the progression of age-related macular degeneration [23]. In addition, similar therapeutic benefits in uveitis mice could be achieved by balancing intestinal Th17/Treg cells through the addition of exogenous SCFAs or its analogs to their diet [68].

FMT is to replace the original gut microbiome of patients with the whole fecal microbiome of healthy donors, so as to change the composition of microorganisms in the gut. FMT can increase the diversity of the microbiome, promote intestinal function, restore bile acid metabolism, and has a significant effect on eradicating $C$. difficile infection in the treatment of colitis [73]. The role of FMT in the treatment of spondyloarthritis and concomitant uveitis was also demonstrated [75]. However, because of differences in the composition of gut microbiome among healthy individuals, it is not clear which individual microbiome is most advantageous for treating a particular disease, and identifying healthy donors for particular diseases may be a challenge for researchers. Besides, the donor materials also need to be strictly tested to avoid posttransplant complications [76].

\section{Conclusion}

The crucial role of intestinal microbiome in the development of noninfectious uveitis has been recently proposed. The major evidences are from animal studies, in which depleting the gut microbiota attenuates disease severity in mouse models of autoimmune uveitis. Clinical studies reported differences in the composition of the microbiota in uveitis patients compared with normal controls. Coinciding with these findings, common immunosuppressant drugs (such as DMARDs) for autoimmune diseases (including uveitis) have some anti-microbial properties. The underlying mechanisms by which the dysbiosis is involved in autoimmune uveitis are not fully understood, but antigenic mimicry, disturbance of intestinal immune homeostasis, destruction of the intestinal barrier, and reduction in beneficial anti-inflammatory metabolites are suggested. Further elucidating these mechanisms is necessary and will facilitate potential therapeutic strategies, including antibiotics, probiotics, diet modifications, and FMT, for the treatment of the gut-microbiome-relevant autoimmune diseases. 


\section{Acknowledgements}

We thank Mr. Fuli Yin for his suggestions for completing our work and manuscript.

\section{Statement of Ethics}

The authors have no ethical conflicts to disclose.

\section{Conflict of Interest Statement}

The authors have no conflicts of interest to declare.

\section{Funding Sources}

This work was supported by grants to DC from the National Natural Science Foundation of China (81870665).

\section{Author Contributions}

D.C. and X.F. made the outline of the manuscript. Y.C. edited the English text. X.F., Y.C., and D.C. wrote and finalized the manuscript.

\section{References}

1 Caspi RR. A look at autoimmunity and inflammation in the eye. J Clin Invest. 2010; 120(9):3073-83.

2 Durrani OM, Tehrani NN, Marr JE, Moradi P, Stavrou P, Murray PI. Degree, duration, and causes of visual loss in uveitis. Br J Ophthalmol. 2004;88(9):1159-62.

3 Forrester JV, Kuffova L, Dick AD. Autoimmunity, autoinflammation, and infection in uveitis. Am J Ophthalmol. 2018;189:77-85.

4 Shimizu J, Kubota T, Takada E, Takai K, Fujiwara N, Arimitsu N, et al. Bifidobacteria abundance-featured gut microbiota compositional change in patients with Behcet's disease. PLoS One. 2016;11(4):e0153746.

5 Horai R, Caspi RR. Microbiome and autoimmune uveitis. Front Immunol. 2019;10:232.

6 Mochizuki M, Sugita S, Kamoi K. Immunological homeostasis of the eye. Prog Retin Eye Res. 2013;33:10-27.

7 Levy M, Kolodziejczyk AA, Thaiss CA, Elinav E. Dysbiosis and the immune system. Nat Rev Immunol. 2017;17(4):219-32.

8 Marchesi JR, Ravel J. The vocabulary of microbiome research: a proposal. Microbiome. 2015;3:31.

9 Szablewski L. Human gut microbiota in health and Alzheimer's disease. J Alzheimers Dis. 2018;62(2):549-60.

10 Sekirov I, Russell SL, Antunes LC, Finlay BB. Gut microbiota in health and disease. Physiol Rev. 2010;90(3):859-904.

11 La Fata G, Rastall R, Lacroix C, Harmsen H, Mohajeri M, Weber P, et al. Recent development of prebiotic research-statement from an expert workshop. Nutrients. 2017;9(12):1376.

12 Whiteside SA, Razvi H, Dave S, Reid G, Burton JP. The microbiome of the urinary tract: a role beyond infection. Nat Rev Urol. 2015; 12(2):81-90.

13 Shivaji S. Connect between gut microbiome and diseases of the human eye. J Biosci. 2019; 44(5): 110.

14 Gritz EC, Bhandari V. The human neonatal gut microbiome: a brief review. Front Pediatr. 2015;3:17.
15 Robles Alonso V, Guarner F. Linking the gut microbiota to human health. Br J Nutr. 2013 109(Suppl 2):S21-6.

16 Ciccia F, Guggino G, Rizzo A, Alessandro R, Luchetti MM, Milling S, et al. Dysbiosis and zonulin upregulation alter gut epithelial and vascular barriers in patients with ankylosing spondylitis. Ann Rheum Dis. 2017;76(6): 1123-32.

17 Wen C, Zheng Z, Shao T, Liu L, Xie Z, Le Chatelier E, et al. Quantitative metagenomics reveals unique gut microbiome biomarkers in ankylosing spondylitis. Genome Biol. 2017; 18(1): 142

18 Gianchecchi E, Fierabracci A. Recent advances on microbiota involvement in the pathogenesis of autoimmunity. Int J Mol Sci. 2019; 20(2):283.

19 Zeng Q, Junli Gong G, Liu X, Chen C, Sun X, $\mathrm{Li} \mathrm{H}$, et al. Gut dysbiosis and lack of short chain fatty acids in a Chinese cohort of patients with multiple sclerosis. Neurochem Int. 2019;129:104468

20 Tsunoda I. Lymphatic system and gut microbiota affect immunopathology of neuroinflammatory diseases, including multiple sclerosis, neuromyelitis optica and Alzheimer's disease. Clin Exp Neuroimmunol. 2017;8(3): 177-9.

21 Kassam F, Gurry T, Aldarmaki A, Nguyen T, Kassam Z, Beck PL, et al. Sa1841: the impact of the gut microbiome in developing uveitis among inflammatory bowel disease patients: a case-control study. Gastroenterology. 2018; 154(6):S-415

22 Barichella M, Severgnini M, Cilia R, Cassani E, Bolliri C, Caronni S, et al. Unraveling gut microbiota in Parkinson's disease and atypical parkinsonism. Mov Disord. 2019;34(3): 396-405.

23 Rowan S, Jiang S, Korem T, Szymanski J, Chang ML, Szelog J, et al. Involvement of a gut-retina axis in protection against dietary glycemia-induced age-related macular degeneration. Proc Natl Acad Sci U S A. 2017; 114(22):E4472-E81.
24 Rinninella E, Mele MC, Merendino N, Cintoni M, Anselmi G, Caporossi A, et al. The role of diet, micronutrients and the gut microbiota in age-related macular degeneration: new perspectives from the gut-retina axis. Nutrients. 2018;10(11).

25 Beli E, Yan Y, Moldovan L, Vieira CP, Gao R, Duan $Y$, et al. Restructuring of the gut microbiome by intermittent fasting prevents retinopathy and prolongs survival in $\mathrm{db} / \mathrm{db}$ mice. Diabetes. 2018;67(9):1867-79.

26 Cavuoto KM, Banerjee S, Galor A. Relationship between the microbiome and ocular health. Ocul Surf. 2019;17(3):384-92.

27 Trujillo-Vargas CM, Schaefer L, Alam J, Pflugfelder SC, Britton RA, de Paiva CS. The gut-eyelacrimal gland-microbiome axis in Sjogren syndrome. Ocul Surf. 2019;18(2):335-44.

28 Doulberis M, Polyzos SA, Papaefthymiou A, Katsinelos P, Kountouras J. Comments to the editor concerning the paper entitled "The microbiome and ophthalmic disease" by Baim et al. Exp Biol Med. 2019;244(6):430-2.

29 Chen H, Cho KS, Vu THK, Shen CH, Kaur M, Chen G, et al. Commensal microflora-induced $\mathrm{T}$ cell responses mediate progressive neurodegeneration in glaucoma. Nat Commun. 2018;9(1):3209.

30 Reháková Z, Capková J, Stěpánková R, Sinkora J, Louzecká A, Ivanyi P, et al. Germ-free mice do not develop ankylosing enthesopathy, a spontaneous joint disease. Hum Immunol. 2000;61(6):555-8

31 Wen L, Ley RE, Volchkov PY, Stranges PB, Avanesyan L, Stonebraker AC, et al. Innate immunity and intestinal microbiota in the development of Type 1 diabetes. Nature. 2008; 455(7216):1109-13.

32 Wu HJ, Ivanov II, Darce J, Hattori K, Shima T, Umesaki Y, et al. Gut-residing segmented filamentous bacteria drive autoimmune arthritis via T helper 17 cells. Immunity. 2010;32(6):815-27.

33 Lee YK, Menezes JS, Umesaki Y, Mazmanian SK. Proinflammatory T-cell responses to gut microbiota promote experimental autoimmune encephalomyelitis. Proc Natl Acad Sc U S A. 2011;108(Suppl 1):4615-22. 
34 Vieira SM, Pagovich OE, Kriegel MA. Diet, microbiota and autoimmune diseases. Lupus. 2014;23(6):518-26.

35 Carding S, Verbeke K, Vipond DT, Corfe BM, Owen LJ. Dysbiosis of the gut microbiota in disease. Microb Ecol Health Dis. 2015;26: 26191.

36 Heissigerova J, Seidler Stangova P, Klimova A, Svozilkova P, Hrncir T, Stepankova R, et al. The microbiota determines susceptibility to experimental autoimmune uveoretinitis. J Immunol Res. 2016;2016:5065703.

37 Nakamura YK, Metea C, Karstens L, Asquith M, Gruner H, Moscibrocki C, et al. Gut microbial alterations associated with protection from autoimmune uveitis. Invest Ophthalmol Vis Sci. 2016;57(8):3747-58.

38 Horai R, Zárate-Bladés CR, Dillenburg-Pilla P, Chen J, Kielczewski JL, Silver PB, et al. Microbiota-dependent activation of an autoreactive $\mathrm{T}$ cell receptor provokes autoimmunity in an immunologically privileged site. Immunity. 2015;43(2):343-53

39 Kim J, Choi SH, Kim YJ, Jeong HJ, Ryu JS, Lee $\mathrm{HJ}$, et al. Clinical effect of IRT-5 probiotics on immune modulation of autoimmunity or alloimmunity in the eye. Nutrients. 2017;9(11): 1166.

40 Huang X, Ye Z, Cao Q, Su G, Wang Q, Deng $\mathrm{J}$, et al. Gut microbiota composition and fecal metabolic phenotype in patients with acute anterior uveitis. Invest Ophthalmol Vis Sci. 2018;59(3):1523-31.

41 Ye Z, Zhang N, Wu C, Zhang X, Wang Q, Huang $X$, et al. A metagenomic study of the gut microbiome in Behcet's disease. Microbiome. 2018;6(1):135.

42 Ye Z, Wu C, Zhang N, Du L, Cao Q, Huang $\mathrm{X}$, et al. Altered gut microbiome composition in patients with Vogt-Koyanagi-Harada disease. Gut Microbes. 2020;11(3):539-17.

43 Kalyana Chakravarthy S, Jayasudha R, Sai Prashanthi G, Ali MH, Sharma S, Tyagi M, et al. Dysbiosis in the gut bacterial microbiome of patients with uveitis, an inflammatory disease of the eye. Indian J Microbiol. 2018 58(4):457-69.

44 Jayasudha R, Kalyana Chakravarthy S, Sai Prashanthi G, Sharma S, Tyagi M, Shivaji S. Implicating dysbiosis of the gut fungal microbiome in uveitis, an inflammatory disease of the eye. Invest Ophthalmol Vis Sci. 2019; 60(5):1384-93.

45 Rossi DC, Ribi C, Guex-Crosier Y. Treatment of chronic non-infectious uveitis and scleritis. Swiss Med Wkly. 2019;149:w20025.

46 Jones NP. Immunosuppression in the management of presumed non-infective uveitis; are we sure what we are treating? Notes on the antimicrobial properties of the systemic immunosuppressants. Ocular Immunolo Inflamm. 2020 Aug 17;28(6):994-1003.

47 Adam L, Phulukdaree A, Soma P. Effective long-term solution to therapeutic remission in inflammatory bowel disease: role of azathioprine. Biomed Pharmacother. 2018;100:814.
48 Tran J, Tsui E. Assessment of the readability, availability, and quality of online patient education materials regarding uveitis medications. Ocul Immunol Inflamm. 2020:1-6.

49 Liu F, Ma R, Riordan SM, Grimm MC, Liu L, Wang Y, et al. Azathioprine, mercaptopurine, and 5-aminosalicylic acid affect the growth of IBD-associated campylobacter species and other enteric microbes. Front Microbiol. 2017;8:527.

50 Antoniani D, Rossi E, Rinaldo S, Bocci P, Lolicato M, Paiardini A, et al. The immunosuppressive drug azathioprine inhibits biosynthesis of the bacterial signal molecule cyclicdi-GMP by interfering with intracellular nucleotide pool availability. Appl Microbiol Biotechnol. 2013;97(16):7325-36.

51 Kruszewska H, Zareba T, Tyski S. Examination of antimicrobial activity of selected nonantibiotic products. Acta Pol Pharm. 2010; 67(6):733-6.

52 Peel M, Scribner A. Cyclophilin inhibitors as antiviral agents. Bioorg Med Chem Lett. 2013; 23(16):4485-92.

53 Kilmartin DJ, Forrester JV, Dick AD. Rescue therapy with mycophenolate mofetil in refractory uveitis. Lancet. 1998;352(9121):35-6.

54 Rojas M, Restrepo-Jiménez P, Monsalve DM, Pacheco Y, Acosta-Ampudia Y, RamírezSantana C, et al. Molecular mimicry and autoimmunity. J Autoimmun. 2018;95:100-23.

55 Avni O, Koren O. Molecular (me)micry? Cell Host Microbe. 2018;23(5):576-8.

56 Miraglia F, Colla E. Microbiome, parkinson's disease and molecular mimicry. Cells. 2019; $8(3): 222$.

57 Zamvil SS, Spencer CM, Baranzini SE, Cree BAC. The gut microbiome in neuromyelitis optica. Neurotherapeutics. 2018;15(1):92101.

58 Varrin-Doyer M, Spencer CM, Schulze-Topphoff U, Nelson PA, Stroud RM, Cree BA, et al. Aquaporin 4-specific T cells in neuromyelitis optica exhibit a Th17 bias and recognize clostridium ABC transporter. Ann Neurol. 2012;72(1):53-64.

59 Cree BA, Spencer CM, Varrin-Doyer M, Baranzini SE, Zamvil SS. Gut microbiome analysis in neuromyelitis optica reveals overabundance of clostridium perfringens. Ann Neurol. 2016;80(3):443-7.

60 Greiling TM, Dehner C, Chen X, Hughes K, Iñiguez $\mathrm{AJ}$, Boccitto $\mathrm{M}$, et al. Commensal orthologs of the human autoantigen Ro60 as triggers of autoimmunity in lupus. Sci Transl Med. 2018;10(434):eaan2306.

61 Collison J. Bacterial orthologues of Ro60 trigger disease. Nat Rev Rheumatol. 2018;14(6): 322.

62 Omenetti S, Pizarro TT. The Treg/Th17 axis: a dynamic balance regulated by the gut microbiome. Front Immunol. 2015;6:639.

63 Zhuang Z, Wang Y, Zhu G, Gu Y, Mao L, Hong M, et al. Imbalance of Th17/Treg cells in pathogenesis of patients with human leukocyte antigen B27 associated acute anterior uveitis. Sci Rep. 2017;7:40414.
64 Leccese P, Alpsoy E. Behçet's disease: an overview of etiopathogenesis. Front Immunol. 2019;10(MAY):1067.

65 Rosenbaum JT, Asquith M. The microbiome and HLA-B27-associated acute anterior uveitis. Nat Rev Rheumatol. 2018;14(12):704-13.

66 Rosenbaum JT, Lin P, Asquith M. The microbiome, HLA, and the pathogenesis of uveitis. Jpn J Ophthalmol. 2016;60(1):1-6.

67 Janowitz C, Nakamura YK, Metea C, Gligor A, Yu W, Karstens L, et al. Disruption of intestinal homeostasis and intestinal microbiota during experimental autoimmune uveitis. Invest Ophthalmol Vis Sci. 2019;60(1):420-9.

68 Nakamura YK, Janowitz C, Metea C, Asquith M, Karstens L, Rosenbaum JT, et al. Short chain fatty acids ameliorate immune-mediated uveitis partially by altering migration of lymphocytes from the intestine. Sci Rep. 2017; 7(1):11745.

69 Ueda Y, Kawakami Y, Kunii D, Okada H, Azuma M, Le DS, et al. Elevated concentrations of linoleic acid in erythrocyte membrane phospholipids in patients with inflammatory bowel disease. Nutr Res. 2008;28(4): 239-44.

70 Ma C, Kesarwala AH, Eggert T, Medina-Echeverz J, Kleiner DE, Jin P, et al. NAFLD causes selective CD4+ T lymphocyte loss and promotes hepatocarcinogenesis. Nature. 2016; 531(7593):253-7.

71 Maes M, Kubera M, Mihaylova I, Geffard M, Galecki P, Leunis JC, et al. Increased autoimmune responses against auto-epitopes modified by oxidative and nitrosative damage in depression: implications for the pathways to chronic depression and neuroprogression. J Affect Disord. 2013;149(1-3):23-9.

72 Morton AM, Sefik E, Upadhyay R, Weissleder R, Benoist C, Mathis D. Endoscopic photoconversion reveals unexpectedly broad leukocyte trafficking to and from the gut. Proc Natl Acad Sci U S A. 2014;111(18):6696-701.

73 Cheng YW, Phelps E, Ganapini V, Khan N, Ouyang F, Xu H, et al. Fecal microbiota transplantation for the treatment of recurrent and severe clostridium difficile infection in solid organ transplant recipients: a multicenter experience. Am J Transplant. 2019;19(2):50111.

74 Lin P. Importance of the intestinal microbiota in ocular inflammatory diseases: a review. Clin Experiment Ophthalmol. 2019;47(3): $418-22$.

75 Choi RY, Asquith M, Rosenbaum JT. Fecal transplants in spondyloarthritis and uveitis: ready for a clinical trial? Curr Opin Rheumatol. 2018;30(4):303-9.

76 Lin P. The role of the intestinal microbiome in ocular inflammatory disease. Curr Opin Ophthalmol. 2018;29(3):261-6.
Gut Microbiome and Autoimmune Uveitis
Ophthalmic Res 2021;64:168-177 